\title{
Potential evolution of neurosurgical treatment paradigms for craniopharyngioma based on genomic and transcriptomic characteristics
}

\author{
Leslie C. Robinson, MD, PharmD, MBA, ${ }^{1}$ Sandro Santagata, MD, PhD, ${ }^{2}$ and \\ Todd C. Hankinson, MD, MBA ${ }^{1}$ \\ ${ }^{1}$ Pediatric Neurosurgery, Children's Hospital Colorado, University of Colorado School of Medicine, Aurora, Colorado; and \\ 2Department of Pathology, Brigham and Women's Hospital, Boston Children's Hospital, Harvard Institute of Medicine, Boston,
} Massachusetts

\begin{abstract}
The recent genomic and transcriptomic characterization of human craniopharyngiomas has provided important insights into the pathogenesis of these tumors and supports that these tumor types are distinct entities. Critically, the insights provided by these data offer the potential for the introduction of novel therapies and surgical treatment paradigms for these tumors, which are associated with high morbidity rates and morbid conditions. Mutations in the CTNNB1 gene are primary drivers of adamantinomatous craniopharyngioma (ACP) and lead to the accumulation of $\beta$-catenin protein in a subset of the nuclei within the neoplastic epithelium of these tumors. Dysregulation of epidermal growth factor receptor (EGFR) and of sonic hedgehog (SHH) signaling in ACP suggest that paracrine oncogenic mechanisms may underlie ACP growth and implicate these signaling pathways as potential targets for therapeutic intervention using directed therapies. Recent work shows that ACP cells have primary cilia, further supporting the potential importance of SHH signaling in the pathogenesis of these tumors. While further preclinical data are needed, directed therapies could defer, or replace, the need for radiation therapy and/or allow for less aggressive surgical interventions. Furthermore, the prospect for reliable control of cystic disease without the need for surgery now exists. Studies of papillary craniopharyngioma (PCP) are more clinically advanced than those for ACP. The vast majority of PCPs harbor the BRAFv600e mutation. There are now 2 reports of patients with $\mathrm{PCP}$ that had dramatic therapeutic responses to targeted agents. Ongoing clinical and research studies promise to not only advance our understanding of these challenging tumors but to offer new approaches for patient management.
\end{abstract}

https://thejns.org/doi/abs/10.3171/2016.9.FOCUS16308

KEY WORDS craniopharyngioma genomics; craniopharyngioma transcriptomics; CTNNB1; BRAF; papillary craniopharyngioma; adamantinomatous craniopharyngioma

$\mathrm{T}$ HE pathobiology of human craniopharyngioma is incompletely understood. This limitation has significantly hindered the ability of clinicians to manage patients afflicted by these tumors, which are associated with high morbidity rates and morbid conditions. The last decade, however, has seen a number of critical discoveries that promise to transform therapeutic approaches. Analyses of the craniopharyngioma genome and transcriptome as well as of the DNA methylation patterns of craniopharyngioma have provided considerable insights into the origins of these tumors and into the drivers of their growth. This work supports that adamantinomatous (ACP) and papillary (PCP) craniopharyngioma are genetically distinct en- tities and has led to the description of tailored therapies for PCP. In this review we discuss the current knowledge regarding the genomic and transcriptomic characteristics of both ACP and PCP and the potential relevance for neurosurgical practice.

\section{Adamantinomatous Craniopharyngioma $\beta$-Catenin and the WNT/Wingless Pathway}

The WNT/wingless pathway contains the only known recurrent genome aberration in ACP. In the normal physiological state, Wnt/wingless signaling is involved in organogenesis and adult stem cell maintenance. ${ }^{26,28}$ Among the

ABBREVIATIONS ACP = adamantinomatous craniopharyngioma; EGF = epidermal growth factor; EGFR = EGF receptor; $\mathrm{PCP}=$ papillary craniopharyngioma; $\mathrm{SHH}=$ sonic hedgehog. 
key members of the canonical WNT signaling pathway is $\beta$-catenin, which is encoded by the CTNNB1 gene. ${ }^{10,32}$ This protein plays a critical role in development, cellular proliferation, differentiation, and cell migration..$^{10,11,16,41} \mathrm{~A}$ destruction complex restrains $\beta$-catenin within the cytosol and facilitates its ubiquitination and proteasomal degradation. ${ }^{31}$ The Wnt protein family includes approximately 20 different proteins that bind to the Frizzled (Fz) family of receptors. When Wnt proteins bind the Fz receptors, the Wnt pathway is activated, ${ }^{31}$ leading to an intracellular signaling cascade that ultimately prevents formation of the $\beta$-catenin destruction complex. ${ }^{9,11,16,22,31}$ Without the destruction complex, $\beta$-catenin protein accumulates and ultimately translocates to the nucleus, where it facilitates transcription of $\beta$-catenin target genes and stimulates cellular proliferation and other Wnt regulated cellular processes. ${ }^{8,9,11,16,22,31}$

Increased migratory capacity and invasiveness have been observed in neoplastic cells with elevated $\beta$-catenin levels and activated target genes..$^{22}$ In ACP, $\beta$-catenin accumulation can be found within cell clusters localized at invasive protrusions of tumor into normal brain ${ }^{22}$ (Fig. 1A). It is possible that this increased mobility and invasive capacity are related to the overexpression of fascin-1 (fascin), a $\beta$-catenin/T-cell factor (TCF) signaling target gene that is commonly involved with reorganization of the actin cytoskeleton, which is required for cellular motility. ${ }^{22,23} \beta$-catenin can regulate fascin expression by binding to a TCF-binding domain on the promoter region for fascin..$^{31}$ Other cancers, such as colorectal, have increased fascin expression, particularly at the invasive edges. ${ }^{22}$ $\beta$-catenin binding of fascin promotes reallocation of $\beta$ catenin, changes cell adhesion properties, and reduces $\beta$ catenin destruction. ${ }^{22}$ As $\beta$-catenin accumulates it can reduce E-cadherin expression, which reduces cell adhesion and likely leads to cells that are more motile and, consequently, more invasive..$^{22}$ Inhibition of fascin or $\beta$-catenin expression can decrease the migratory capacity of ACP tumor cells in culture. ${ }^{22}$

\section{ACP Genome Analyses \\ CTNNB1 Mutation}

Nuclear accumulation of $\beta$-catenin results from mutations within exon 3 of the CTNNB1 gene. This exon encodes a degradation targeting motif, mutation of which confers resistance to the $\beta$-catenin destruction complex., ${ }^{9,41}$ While mutations have been identified at a number of different codons, these all impact the binding of GSK3 $\beta .9,11,12,24,28,38$ As a result of this mechanism, nuclear accumulation of $\beta$-catenin is a histological hallmark of CTNNB1 mutation. ${ }^{10,12,16,26,41}$ In the case of craniopharyngiomas, mutation of $C T N N B 1$ has been described in $69 \%-100 \% 9,20,28$ of ACP specimens, while it is not routinely observed in PCP or other parasellar tumors. ${ }^{9,28}$ In the most detailed study to date, CTNNB1 mutation was identified in $95 \%$ of ACP specimens, using massively parallel sequencing and targeted genotyping. ${ }^{5}$ In the same study, Brastianos and Santagata determined that $C T N N B 1$ mutation was not present in PCP. ${ }^{5}$ Other groups have identified CTNNB1 mutation in 70\%-80\% of ACP specimens..$^{9,12,19,31}$ Sampling error or
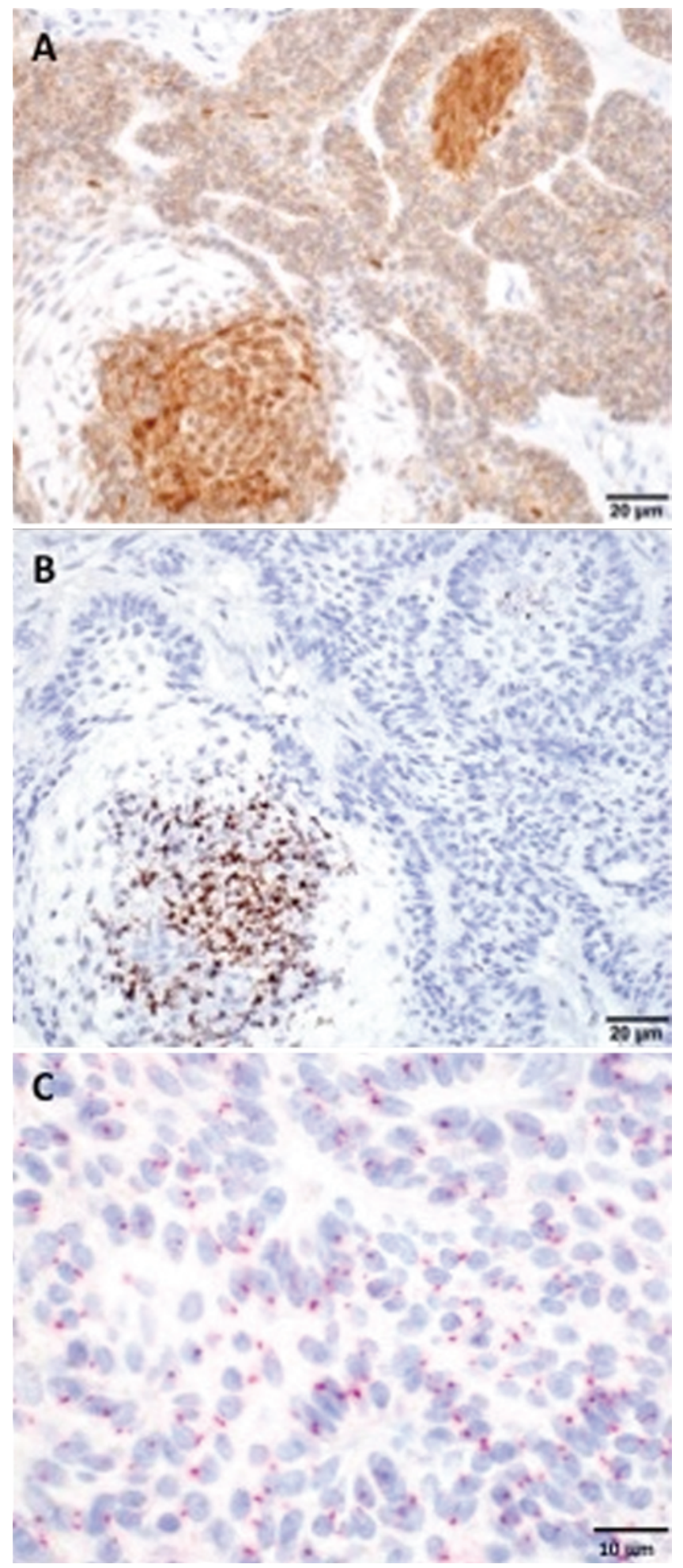

FIG. 1. A: Immunohistochemistry for $\beta$-catenin demonstrates nuclear and cytoplasmic staining in clusters of tumor cells in human ACP. B: In situ hybridization shows a high level of expression of SHH mRNA in areas with nuclear $\beta$-catenin. C: Immunohistochemistry for the cilia marker ARL13B shows primary cilia in the majority of tumor cells in ACP. 
the significant intratumoral heterogeneity present in these tumors, which often have scant neoplastic epithelium, may explain the variability in the frequency with which CTNNB1 mutation is detected. Presently, however, despite the presence of $\beta$-catenin dysregulation in multiple cancer cell types, no targeted therapies are available. As such, direct intervention regarding $C T N N B 1$ mutation is unlikely to impact neurosurgical treatment algorithms in the short term.

Nevertheless, insights related to the mutation affecting the GSK $3 \beta$ binding domain of $C T N N B 1$ have implications regarding the origins of ACP. A clearer understanding of these may facilitate efforts to identify directed therapies. For example, definitive evidence of pituitary origin would indicate that ACP is not protected by the blood-brain barrier, thereby suggesting that a wider range of systemically delivered antitumor therapies could be effective. While similar mutations are observed in other lesions that share histological features with ACP, such as pilomatricoma and calcifying cystic odontogenic tumor, ${ }^{21}$ multiple potential origins for ACP remain to be explored. As mentioned above, it has been postulated that ACP arises from undifferentiated anterior pituitary stem cells. ${ }^{17,20,23,39}$ An additional potential mechanism employs the paracrine tumorigenesis theory, in which small clusters of CTNNB1 mutated cells, characterized by nucleocytoplasmic $\beta$-catenin, leverage paracrine/autocrine signaling to promote tumorigenic activity in a much larger nonmutated cell population. 7,35

\section{The Sonic Hedgehog Pathway}

Sonic hedgehog $(\mathrm{SHH})$ plays an integral role in the maintenance of adult stem cells ${ }^{2}$ and in the normal development of several organs, including the pituitary gland and Rathke's pouch. ${ }^{18,31}$ Pathological upregulation of SHH signaling has been demonstrated in a multitude of cancers, including meningioma, basal cell carcinoma, and medulloblastoma. ${ }^{1,2,4,14,29,40}$ With regard to ACP, SHH overexpression has been described in tumors in humans and in a novel murine model, in which it colocalizes in cells with nuclear accumulation of $\beta$-catenin (Fig. 1B). ${ }^{2}$ It is hypothesized that both autocrine and paracrine SHH signaling may contribute to ACP tumorigenesis. Two studies of the human ACP transcriptome have shown an upregulation of SHH pathway genes ${ }^{18,20}$ (Fig. 2). The therapeutic relevance of this finding was solidified by the demonstration that ACP uniquely expressed the active isoform of SHH protein, when compared with other pediatric brain tumor types and to normal brain. ${ }^{20}$ As such, preclinical animal studies of the smoothened inhibitor vismodegib are ongoing (personal communication, J. P. Martinez-Barbera, 2016).

An additional application of novel insights regarding the SHH pathway in craniopharyngioma lies in the differentiation of ACP from PCP. Hölsken and colleagues ${ }^{25}$ demonstrated a much higher expression of SHH pathway genes in the transcriptomes of ACP compared with PCP. These findings correlated with distinct methylation patterns between the 2 tumor types. This again suggests that ACP and PCP are biologically distinct tumors. Interestingly, among ACP specimens, the authors identified no difference in the methylation pattern of tumors based on the patient's age at presentation. This implies that therapies identified through the study of pediatric tumors may also have relevance in the adult setting. The distinction between ACP and PCP was further supported by a recent study showing different patterns of primary cilia between these lesions. ${ }^{15}$ Using immunohistochemistry for ARL13B, Coy and colleagues showed that ACPs have primary cilia throughout their neoplastic epithelium (Fig. 1C), whereas PCPs have primary cilia only in the basal cells of the neoplastic epithelium. Because neoplasms that are driven by oncogenic activation of the hedgehog pathway, such as basal cell carcinoma, and a subset of medulloblastomas often retain primary cilia and require these organelles for hedgehog signaling, the identification of primary cilia throughout the epithelium of ACP further supports the potential importance of SHH signaling and perhaps other cilia-dependent signaling pathways in the pathogenesis of ACP.15

\section{Epidermal Growth Factor Receptor}

There does not appear to be genome-level dysregulation of the epidermal growth factor (EGF) pathway in ACP. However, downstream upregulation of this pathway may contribute to ACP cell growth and infiltration, and offers the prospect of effective targeted therapies that could influence the role of neurosurgical intervention for patients with ACP. The EGF receptor (EGFR) is a transmembrane glycoprotein that can be activated by multiple growth factors, resulting in the initiation of intracellular cascades involved in the regulation of cell proliferation, differentiation, apoptosis, and motility. ${ }^{23}$ There is evidence that, when activated and phosphorylated, EGFR and $\beta$-catenin colocalize to the nucleus, along with fascin. As described above, this occurs in cell clusters at the infiltrating borders of the tumor, in which the canonical Wnt pathway is upregulated..$^{23,31,33}$ Correlation between increased EGFR pathway activity and ACP cell growth and migration has been seen in laboratory data ${ }^{16,20}$ and cell culture models. ${ }^{23}$ Additional evidence supporting the role of EGFR in tumorigenesis of ACP includes high levels of EGF pathway genes in the ACP transcriptome,$^{20}$ the involvement of EGFR in the regulation of the expression of stem cell markers in ACP, and the presence of the activated EGFR pathway in $\beta$-catenin accumulating cells. ${ }^{26}$ As with therapies directed at the SHH pathway, those targeting the EGF pathway require further clarification through preclinical study before they could enter clinical use and impact neurosurgical care.

\section{Additional Relevant Pathways}

In addition to SHH and EGFR pathways, other molecules and pathways are uniquely expressed in craniopharyngioma, but are not yet as well described. The ACP transcriptome is characterized by high levels of certain matrix metallopeptidases, ${ }^{19,20}$ including MMPs 2, 3, 7, 9, and 12. Elevated levels of p63 have been found in both ACP and PCP, with elevated expression also identified in the ACP transcriptome..$^{20} \mathrm{P} 63$ is a gene homolog of the p53 tumor suppressor family. ${ }^{12,16}$ Impaired p63 expression has been suggested to influence neoplastic cell transformation. ${ }^{12}$ In addition, transcriptome analysis identified significant overexpression-in ACP - of LCK, EPHA, and $\mathrm{SRC}$, each of which is a target of the tyrosine kinase inhib- 

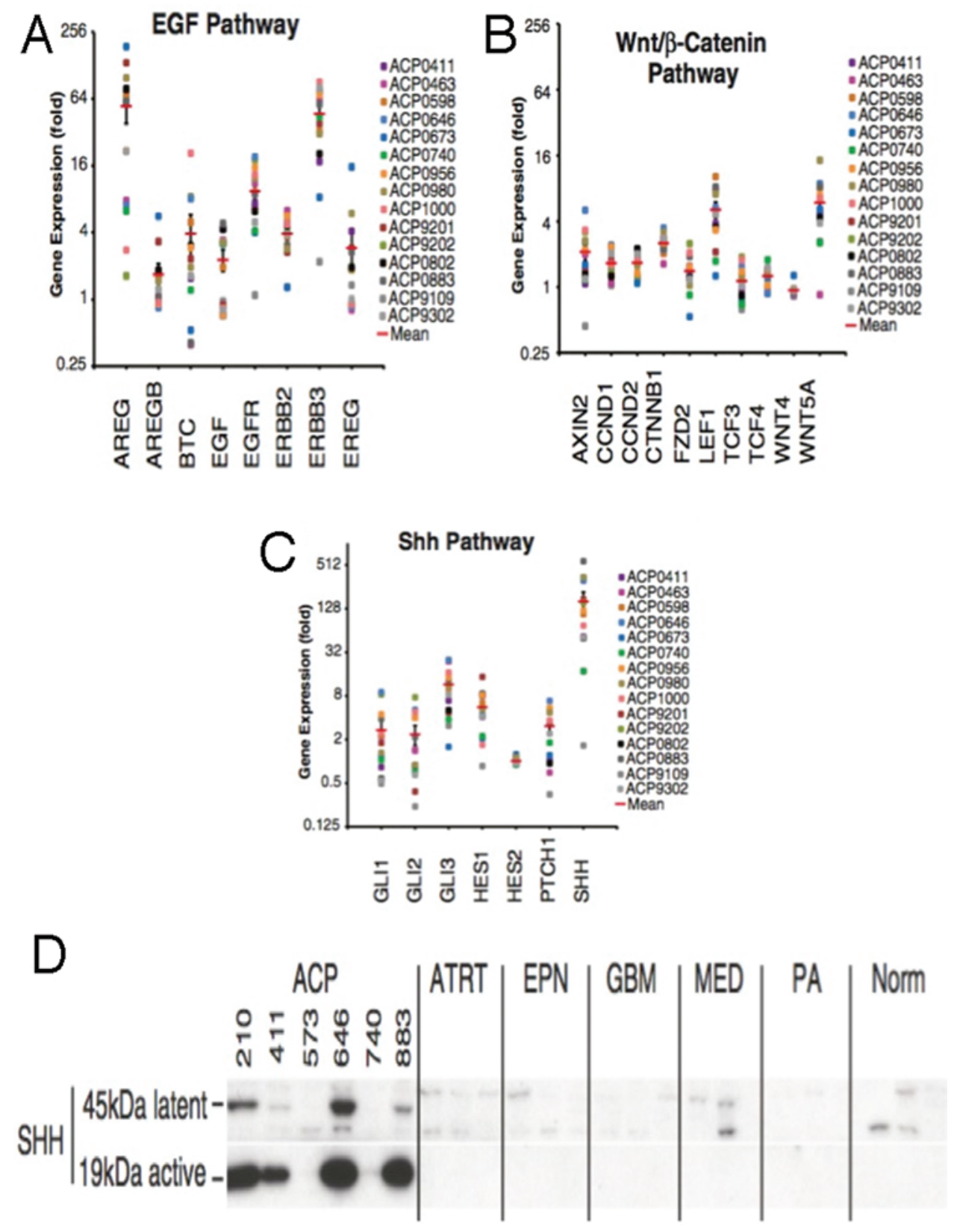

FIG. 2. A-C: Expression of the indicated developmental and cancer-related genes in individual ACP samples: EGF genes (A), WNT pathway (B), and SHH pathway (C). D: Western blot analysis demonstrating overexpression of the latent preforms and active cleaved protein isoforms of SHH in ACP relative to other common pediatric brain tumors and normal brain. ATRT = atypical teratoid/rhabdoid tumor; EPN = ependymoma; GBM = glioblastoma; $\mathrm{MED}=$ medulloblastoma; Norm = normal brain; $\mathrm{PA}=$ pilocytic astrocytoma. Reproduced with slight modification from Gump et al: Acta Neuropathol Commun 3:30, 2015; CC BY 4.0 (https:// creativecommons.org/licenses/by/4.0/).

itor dasatinib. ${ }^{20}$ While still in the preclinical stages, these unique characteristics of ACP may at some point allow for effective therapeutic intervention without radical surgery.

\section{Neurosurgical Implications of ACP}

While current therapy for ACP relies upon surgery, radiation, or cyst-directed intervention, the biological characteristics described above hold promise for the introduction of targeted antitumor treatments. The details of therapy delivery, such as the timing and route (e.g., sys- temic, intrathecal), and therefore the precise role of operative intervention, remain to be determined. Presently, preclinical studies to identify the paradigms with the highest likelihood of success are ongoing.

\section{Papillary Craniopharyngioma \\ Distinction From ACP}

As described above, ACP and PCP demonstrate distinct genetic aberrations. Different transcriptome profiles 


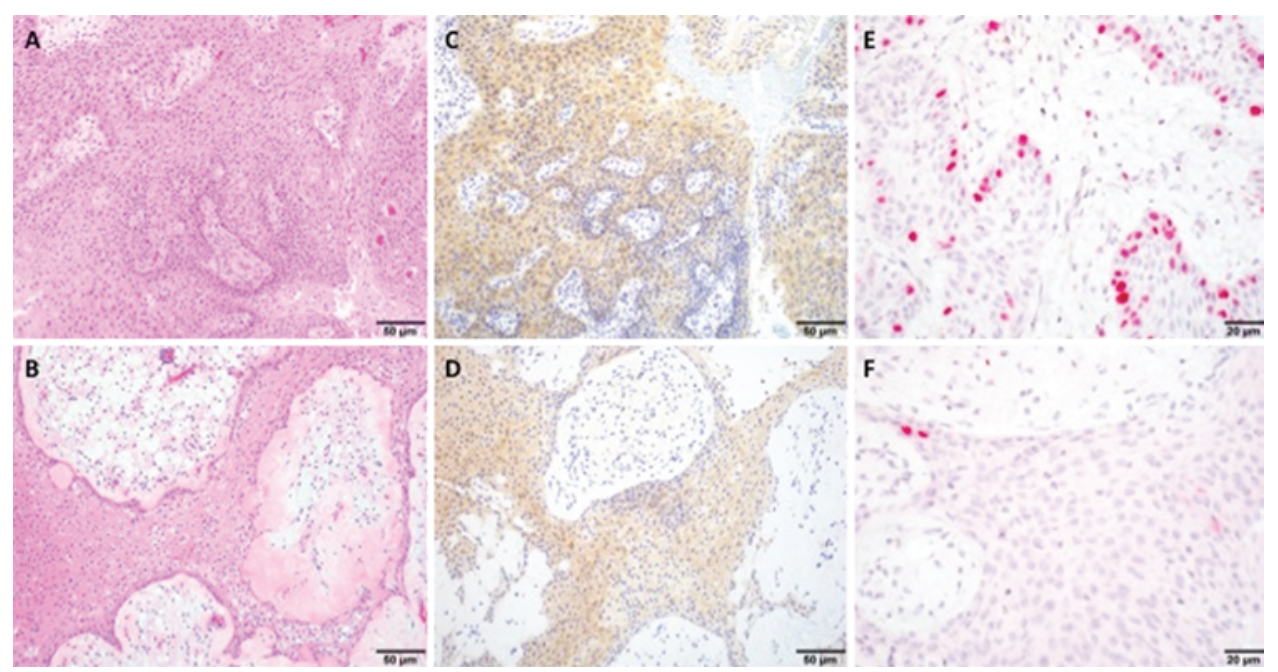

FIG. 3. Effect of dual therapy using dabrafenib and trametinib on multiply recurrent PCP. H \& E-stained specimens prior to (A) and $\operatorname{after}(\mathrm{B})$ treatment. Immunohistochemistry that recognizes BRAF V600E mutant protein specifically (VE1 clone) shows that the $B R A F$ mutation is present in the tumor epithelium in both the pretreatment (C) and posttreatment (D) specimens, thereby demonstrating that the treatment did not entirely eradicate tumor cells. Treatment with dabrafenib and trametinib results in a marked decrease in the number of cycling cells (MIB-1 immunohistochemistry on pretreatment [E] and posttreatment [F] samples).

and differences in genome-wide methylation patterns support the view that these 2 tumors are biologically distinct entities with different mechanisms of pathogenesis. This distinction has substantial implications for neurosurgeons, as case reports of successful directed therapy for PCP, derived through experience with more common tumors, have been published. Successful clinical trials of these treatments could alter the role of the neurosurgeon in the management of PCP. Genomic studies support the findings from histological studies that show nuclear and cytoplasmic accumulation of $\beta$-catenin in ACP. In PCP, $\beta$-catenin localizes to the cell membrane, similar to the pattern of localization in other CTNNB1 wild-type tumors of the sellar region ${ }^{9,12,16,31}$ and throughout the body.

\section{BRAF}

Mutation of the $B R A F$ proto-oncogene is well characterized in the context of multiple human cancers, making it the most clinically relevant genome-level aberration that has been identified in human craniopharyngioma. $B R A F$ mutation upregulates MAP kinase signaling, thereby driving cell division. Although multiple mutations of $B R A F$ have been described in human cancers, the most common is a substitution of valine by glutamate at codon number 600 , termed the $B R A F^{\mathrm{v} 600 \mathrm{e}}$ mutation. The work of Brastianos and colleagues, discussed above, ${ }^{7}$ identified $B R A F^{\mathrm{v} 600 \mathrm{e}}$ mutation in $92.8 \%$ of PCP specimens. This mutation was not found in any of the ACP specimens examined. This raised the potential for effective targeted therapy using medications that were developed in the context of other tumors that harbor $B R A F$ mutation, most notably cutaneous melanoma. In this report, however, whole-exome sequencing was undertaken in only 3 PCP specimens. As such, the existence of additional lower frequency recurrent genomic events remains a possibility. Nevertheless, multiple studies demonstrate the presence of $B R A F^{\mathrm{v} 600 \mathrm{e}}$ mutation in nearly every specimen of confirmed PCP..$^{30,36,37}$ An additional intriguing report suggests that a small subset of human craniopharyngioma is characterized by coexisting mutations in both CTNNBI and BRAF.32 This could be of substantial clinical interest and merits further exploration, but presently does not impact neurosurgical treatment strategies.

Leveraging the presence of the $B R A F^{\mathrm{v} 600 \mathrm{e}}$ mutation, 2 recent case reports demonstrated clinically relevant responses of PCP to directed therapies. Aylwin and colleagues $^{3}$ described a near-total radiographic response of a $B R A F^{\mathrm{v} 600 \mathrm{e}}$ mutant PCP following treatment with single agent vemurafenib, a mutation-specific BRAF inhibitor. Importantly, after therapy was halted for a CSF leak associated with tumor shrinkage, tumor recurrence occurred within 6 weeks and long-term control was not achieved after therapy was restarted. In a 39-year-old patient with multiply recurrent PCP, who had required 4 craniotomies and an endonasal resection for multiple cystic recurrences, Brastianos and collegues ${ }^{6}$ reported an excellent initial response to combination therapy using the RAF inhibitor dabrafenib and MEK inhibitor trametinib (Fig. 3). Furthermore, the authors identified $B R A F^{v 600 e}$ mutant DNA in the patient's peripheral blood during therapy, thus presenting the prospect of noninvasive diagnosis for patients with suspected PCP. These reports highlight the facts that multiple directed therapies are potentially available for patients with PCP and that dual or multiagent therapy is likely to be required to reliably obtain durable tumor control. As such, a multi-institutional trial of combined BRAF and MEK inhibition for patients with PCP is forthcoming. ${ }^{5}$

In addition to offering therapeutic potential, the high prevalence of the $B R A F^{\mathrm{v} 600 \mathrm{e}}$ mutation in PCP may simplify diagnosis of these tumors. A mutation-specific antibody (clone VE1) recognizes the BRAFv600e mutant protein but not the wild-type protein. ${ }^{13}$ This is now being employed by pathologists for immunohistochemical identification, thereby distinguishing PCP from other masses 
of the sellar region, including pituitary adenomas, ACP, and Rathke's cleft cysts with squamous metaplasia. ${ }^{27,30,37}$ Targeted genotyping of the $B R A F^{\mathrm{v} 600 \mathrm{e}}$ mutant allele is also used for diagnosis and other biomarkers have been proposed as well. ${ }^{34}$

\section{Neurosurgical Implications of PCP}

For neurosurgeons, the potential impact of recent advances in our biological understanding of $\mathrm{PCP}$, as well as noninvasive identification of BRAF mutated cells, is considerable. Neoadjuvant systemic therapy that does not necessitate aggressive (or any) surgical intervention could assume a prominent position in the treatment paradigm for patients with PCP. While PCP represents the minority of craniopharyngiomas, and is almost exclusively limited to the adult population, this represents dramatic progress in the clinical management of this tumor associated with high morbidity.

\section{Conclusions}

Prospects for effective novel therapies against craniopharyngiomas in humans have improved considerably with recent advances in our understanding of the biological basis of this disease. Genomic and transcriptomic analyses indicate that $\mathrm{ACP}$ and $\mathrm{PCP}$ will require different therapeutic approaches. Promising options for PCP have emerged from previous experience with more common tumors that harbor the $B R A F^{\mathrm{v} 600 \mathrm{e}}$ mutation, and human clinical trials will further investigate these advances. These trials may allow neurosurgeons to reserve aggressive intervention, with the associated morbidity, for the minority of cases that do not demonstrate a durable response to directed therapies. Although we currently lack therapies that target the $\beta$-catenin mutation that characterizes ACP, multiple additional targets, such as the SHH and EGFR pathways, have been described. Further preclinical investigation of these prospects is ongoing and may ultimately, as in the case of PCP, influence neurosurgical management paradigms.

\section{References}

1. Abedalthagafi M, Bi WL, Aizer AA, Merrill PH, Brewster R, Agarwalla PK, et al: Oncogenic PI3K mutations are as common as AKT1 and SMO mutations in meningioma. Neuro Oncol 18:649-655, 2016

2. Andoniadou CL, Gaston-Massuet C, Reddy R, Schneider RP, Blasco MA, Le Tissier P, et al: Identification of novel pathways involved in the pathogenesis of human adamantinomatous craniopharyngioma. Acta Neuropathol 124:259-271, 2012

3. Aylwin SJ, Bodi I, Beaney R: Pronounced response of papillary craniopharyngioma to treatment with vemurafenib, a BRAF inhibitor. Pituitary 19:544-546, 2016

4. Brastianos PK, Horowitz PM, Santagata S, Jones RT, McKenna A, Getz G, et al: Genomic sequencing of meningiomas identifies oncogenic SMO and AKT1 mutations. Nat Genet 45:285-289, 2013

5. Brastianos PK, Santagata S: Endocrine tumors: BRAF V600E mutations in papillary craniopharyngioma. Eur $\mathbf{J}$ Endocrinol 174:R139-R144, 2016

6. Brastianos PK, Shankar GM, Gill CM, Taylor-Weiner A, Nayyar N, Panka DJ, et al: Dramatic response of BRAF
V600E mutant papillary craniopharyngioma to targeted therapy. J Natl Cancer Inst 108:108, 2015

7. Brastianos PK, Taylor-Weiner A, Manley PE, Jones RT, DiasSantagata D, Thorner AR, et al: Exome sequencing identifies BRAF mutations in papillary craniopharyngiomas. Nat Genet 46:161-165, 2014

8. Buslei R, Hölsken A, Hofmann B, Kreutzer J, Siebzehnrubl F, Hans V, et al: Nuclear $\beta$-catenin accumulation associates with epithelial morphogenesis in craniopharyngiomas. Acta Neuropathol 113:585-590, 2007

9. Buslei R, Nolde M, Hofmann B, Meissner S, Eyupoglu IY, Siebzehnrübl F, et al: Common mutations of $\beta$-catenin in adamantinomatous craniopharyngiomas but not in other tumours originating from the sellar region. Acta Neuropathol 109:589-597, 2005

10. Campanini ML, Colli LM, Paixao BM, Cabral TP, Amaral FC, Machado HR, et al: CTNNB1 gene mutations, pituitary transcription factors, and MicroRNA expression involvement in the pathogenesis of adamantinomatous craniopharyngiomas. Horm Cancer 1:187-196, 2010

11. Cani CMG, Matushita H, Carvalho LRS, Soares IC, Brito LP, Almeida MQ, et al: PROP1 and CTNNB1 expression in adamantinomatous craniopharyngiomas with or without $\beta$ catenin mutations. Clinics (Sao Paulo) 66:1849-1854, 2011

12. Cao J, Lin JP, Yang LX, Chen K, Huang ZS: Expression of aberrant $\beta$-catenin and impaired p63 in craniopharyngiomas. Br J Neurosurg 24:249-256, 2010

13. Capper D, Preusser M, Habel A, Sahm F, Ackermann U, Schindler G, et al: Assessment of BRAF V600E mutation status by immunohistochemistry with a mutation-specific monoclonal antibody. Acta Neuropathol 122:11-19, 2011

14. Clark VE, Erson-Omay EZ, Serin A, Yin J, Cotney J, Ozduman K, et al: Genomic analysis of non-NF2 meningiomas reveals mutations in TRAF7, KLF4, AKT1, and SMO. Science 339: 1077-1080, 2013

15. Coy S, Du Z, Sheu SH, Woo T, Rodriguez FJ, Kieran MW, et al: Distinct patterns of primary and motile cilia in Rathke's cleft cysts and craniopharyngioma subtypes. Mod Pathol [epub ahead of print], 2016

16. Esheba GE, Hassan AA: Comparative immunohistochemical expression of $\beta$-catenin, EGFR, ErbB2, and p63 in adamantinomatous and papillary craniopharyngiomas. J Egypt Natl Canc Inst 27:139-145, 2015

17. Gaston-Massuet C, Andoniadou CL, Signore M, Jayakody SA, Charolidi N, Kyeyune R, et al: Increased Wingless (Wnt) signaling in pituitary progenitor/stem cells gives rise to pituitary tumors in mice and humans. Proc Natl Acad Sci U S A 108:11482-11487, 2011

18. Gomes DC, Jamra SA, Leal LF, Colli LM, Campanini ML, Oliveira RS, et al: Sonic Hedgehog pathway is upregulated in adamantinomatous craniopharyngiomas. Eur J Endocrinol 172:603-608, 2015

19. Gong J, Zhang H, Xing S, Li C, Ma Z, Jia G, et al: High expression levels of CXCL12 and CXCR4 predict recurrence of adamanti-nomatous craniopharyngiomas in children. Cancer Biomark 14:241-251, 2014

20. Gump JM, Donson AM, Birks DK, Amani VM, Rao KK, Griesinger AM, et al: Identification of targets for rational pharmacological therapy in childhood craniopharyngioma. Acta Neuropathol Commun 3:30, 2015

21. Hassanein AM, Glanz SM, Kessler HP, Eskin TA, Liu C: $\beta$ Catenin is expressed aberrantly in tumors expressing shadow cells. Pilomatricoma, craniopharyngioma, and calcifying odontogenic cyst. Am J Clin Pathol 120:732-736, 2003

22. Hölsken A, Buchfelder M, Fahlbusch R, Blümcke I, Buslei R: Tumour cell migration in adamantinomatous craniopharyngiomas is promoted by activated Wnt-signalling. Acta Neuropathol 119:631-639, 2010

23. Hölsken A, Gebhardt M, Buchfelder M, Fahlbusch R, 
Blümcke I, Buslei R: EGFR signaling regulates tumor cell migration in craniopharyngiomas. Clin Cancer Res 17:4367-4377, 2011

24. Hölsken A, Kreutzer J, Hofmann BM, Hans V, Oppel F, Buchfelder M, et al: Target gene activation of the Wnt signaling pathway in nuclear $\beta$-catenin accumulating cells of adamantinomatous craniopharyngiomas. Brain Pathol 19:357-364, 2009

25. Hölsken A, Sill M, Merkle J, Schweizer L, Buchfelder M, Flitsch J, et al: Adamantinomatous and papillary craniopharyngiomas are characterized by distinct epigenomic as well as mutational and transcriptomic profiles. Acta Neuropathol Commun 4:20, 2016

26. Hölsken A, Stache C, Schlaffer SM, Flitsch J, Fahlbusch R, Buchfelder M, et al: Adamantinomatous craniopharyngiomas express tumor stem cell markers in cells with activated Wnt signaling: further evidence for the existence of a tumor stem cell niche? Pituitary 17:546-556, 2014

27. Jones RT, Abedalthagafi MS, Brahmandam M, Greenfield EA, Hoang MP, Louis DN, et al: Cross-reactivity of the BRAF VE1 antibody with epitopes in axonemal dyneins leads to staining of cilia. Mod Pathol 28:596-606, 2015

28. Kato K, Nakatani Y, Kanno H, Inayama Y, Ijiri R, Nagahara $\mathrm{N}$, et al: Possible linkage between specific histological structures and aberrant reactivation of the Wnt pathway in adamantinomatous craniopharyngioma. J Pathol 203:814-821, 2004

29. Katoh Y, Katoh M: Hedgehog target genes: mechanisms of carcinogenesis induced by aberrant hedgehog signaling activation. Curr Mol Med 9:873-886, 2009

30. Kim JH, Paulus W, Heim S: BRAF V600E mutation is a useful marker for differentiating Rathke's cleft cyst with squamous metaplasia from papillary craniopharyngioma. J Neurooncol 123:189-191, 2015

31. Larkin SJ, Ansorge O: Pathology and pathogenesis of craniopharyngiomas. Pituitary 16:9-17, 2013

32. Larkin SJ, Preda V, Karavitaki N, Grossman A, Ansorge $\mathrm{O}$ : BRAF V600E mutations are characteristic for papillary craniopharyngioma and may coexist with CTNNB1-mutated adamantinomatous craniopharyngioma. Acta Neuropathol 127:927-929, 2014

33. Lee CH, Hung HW, Hung PH, Shieh YS: Epidermal growth factor receptor regulates $\beta$-catenin location, stability, and transcriptional activity in oral cancer. Mol Cancer 9:64, 2010

34. Liu Y, Wang CH, Li DL, Zhang SC, Peng YP, Peng JX, et al. TREM-1 expression in craniopharyngioma and Rathke's cleft cyst: its possible implication for controversial pathology. Oncotarget [epub ahead of print], 2016

35. Martinez-Barbera JP, Andoniadou CL: Concise review: Para- crine role of stem cells in pituitary tumors: a focus on adamantinomatous craniopharyngioma. Stem Cells 34:268-276, 2016

36. Marucci G, de Biase D, Zoli M, Faustini-Fustini M, Bacci A, Pasquini E, et al: Targeted BRAF and CTNNB1 next-generation sequencing allows proper classification of nonadenomatous lesions of the sellar region in samples with limiting amounts of lesional cells. Pituitary 18:905-911, 2015 (Erratum in Pituitary 19:113, 2016)

37. Schweizer L, Capper D, Hölsken A, Fahlbusch R, Flitsch J, Buchfelder M, et al: BRAF V600E analysis for the differentiation of papillary craniopharyngiomas and Rathke's cleft cysts. Neuropathol Appl Neurobiol 41:733-742, 2015

38. Sekine S, Shibata T, Kokubu A, Morishita Y, Noguchi M, Nakanishi Y, et al: Craniopharyngiomas of adamantinomatous type harbor $\beta$-catenin gene mutations. Am J Pathol 161:1997-2001, 2002

39. Sekine S, Takata T, Shibata T, Mori M, Morishita Y, Noguchi $\mathrm{M}$, et al: Expression of enamel proteins and LEF1 in adamantinomatous craniopharyngioma: evidence for its odontogenic epithelial differentiation. Histopathology 45:573-579, 2004

40. Taylor MD, Northcott PA, Korshunov A, Remke M, Cho YJ, Clifford SC, et al: Molecular subgroups of medulloblastoma: the current consensus. Acta Neuropathol 123:465-472, 2012

41. Zuhur SS, Tanik C, Erol RS, Musluman AM, Kabukcuoglu F, Altuntas Y: Immunohistochemical expression of ErbB2 in adamantinomatous craniopharyngiomas: a possible target for immunotherapy. Turk Neurosurg 23:55-60, 2013

\section{Disclosures}

The authors report no conflict of interest concerning the materials or methods used in this study or the findings specified in this paper.

\section{Author Contributions}

Conception and design: all authors. Acquisition of data: all authors. Analysis and interpretation of data: all authors. Drafting the article: all authors. Critically revising the article: all authors. Reviewed submitted version of manuscript: all authors. Approved the final version of the manuscript on behalf of all authors: Hankinson.

\section{Correspondence}

Todd C. Hankinson, Department of Pediatric Neurosurgery, Children's Hospital Colorado, 13123 E 16th Ave., Box 330, Aurora, CO 80045. email: todd.hankinson@childrenscolorado.org. 